Article

\title{
Evaluation of Nystatin Containing Chitosan Hydrogels as Potential Dual Action Bio-Active Restorative Materials: in Vitro Approach
}

\author{
V. Tamara Perchyonok ${ }^{1, *}$, Vanessa Reher ${ }^{2}$, Shengmiao Zhang ${ }^{4}$, Nicki Basson ${ }^{3}$ \\ and Sias Grobler ${ }^{3}$
}

1 Research and Development Department, VTPCHEM PTY LTD., Southport 4215, Australia

2 School of Dentistry and Oral Health, Gold Coast campus, Griffith University, QLD 4222, Australia

3 Oral and Dental Research Institute, Faculty of Dentistry, University of the Western Cape, Private Bag X1, Tygerberg 7505, Cape Town, South Africa

4 School of Material Science and Engineering, East China University of Science and Technology, 130 Meilong Road, Shanghai 200237, China

* Author to whom correspondence should be addressed; E-Mail: tamaraperchyonok@gmail.com; Tel.: +61-414-596-304.

External Editor: Francesco Puoci

Received: 29 August 2014; in revised form: 27 October 2014 / Accepted: 24 November 2014 / Published: 28 November 2014

\begin{abstract}
Healing is a specific biological process related to the general phenomenon of growth and tissue regeneration and is a process generally affected by several systemic conditions or as detrimental side-effects of chemotherapy- and radiotherapy-induced inflammation of the oral mucosa. The objectives of this study is to evaluate the novel chitosan based functional drug delivery systems, which can be successfully incorporated into "dual action bioactive restorative materials", capable of inducing in vitro improved wound healing prototype and containing an antibiotic, such as nystatin, krill oil as an antioxidant and hydroxyapatite as a molecular bone scaffold, which is naturally present in bone and is reported to be successfully used in promoting bone integration when implanted as well as promoting healing. The hydrogels were prepared using a protocol as previously reported by us. The physico-chemical features, including surface morphology (SEM), release behaviors, stability of the therapeutic agent-antioxidant-chitosan, were measured and compared to the
\end{abstract}


earlier reported chitosan-antioxidant containing hydrogels. Structural investigations of the reactive surface of the hydrogel are reported. Release of nystatin was investigated for all newly prepared hydrogels. Bio-adhesive studies were performed in order to assess the suitability of these designer materials. Free radical defense capacity of the biomaterials was evaluated using established in vitro model. The bio-adhesive capacity of the materials in the in vitro system was tested and quantified. It was found that the favorable synergistic effect of free radical built-in defense mechanism of the new functional materials increased sustainable bio-adhesion and therefore acted as a functional multi-dimensional restorative material with potential application in wound healing in vitro.

Keywords: chitosan; hydrogel; nystatin; reactive oxygen species; antioxidants; functional biomaterials; microbiological activity; percentage release

\section{Introduction}

Reactive oxygen species (ROS) are associated with all the stages of the healing process [1-6]. ROS are produced by the inflammatory cells and play an integral role during this process [7-12]. Antioxidants administration is beneficial for healing [13].

Bioadhesive polymers appear to be particularly attractive for the development of alternative etch free dentin bonding system with an added advantage of additional therapeutic delivery systems to improve intra-dental administration of therapeutic and prophylactic agents if necessary [10-15]. Chitosan, which is a biologically safe biopolymer, has been proposed as a bioadhesive polymer and are of continuous interest to us due to its unique properties and flexibility in a broad range of oral applications reported by others and us recently [16-20].

The objectives of this study is to evaluate the novel chitosan based functional drug delivery systems, which can be successfully incorporated into "dual action bioactive restorative materials" capable to induce in vitro improved wound healing prototype and containing common antibiotics, such as nystatin, krill oil as an antioxidant, hydroxyapatite as a molecular bone scaffold, which is naturally present in bone and is reported to be successfully used in promoting bone integration when implanted as well as promoting healing.

\section{Results and Discussion}

The SEM images were obtained to characterize the microstructure of the freeze-dried gels and are presented in Figure 1. It could be seen that the gels displayed a homogeneous pore structure similar to a sponge. SEM analysis revealed interconnected pores of different sizes and flat, relatively smooth walls. The biomaterial remained intact after 24 days of immersion in artificial saliva as was confirmed by SEM. It was thought that the micro-porous structure of the gels could lead to high internal surface areas with low diffusional resistance in the gels. The $\mathrm{pH}$ of the prepared gels ranged from 5.46 to 6.94 (Table 1). 
Figure 1. SEM photomicrograph of freeze-dried gels 1-5 (a-e).

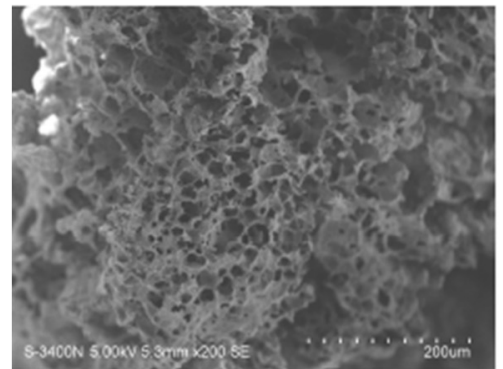

a

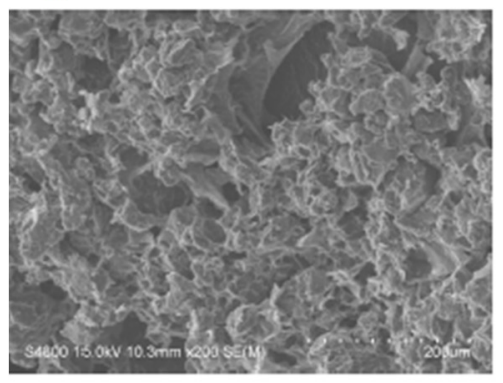

d

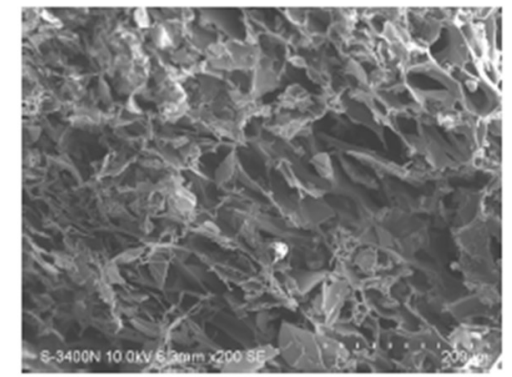

b

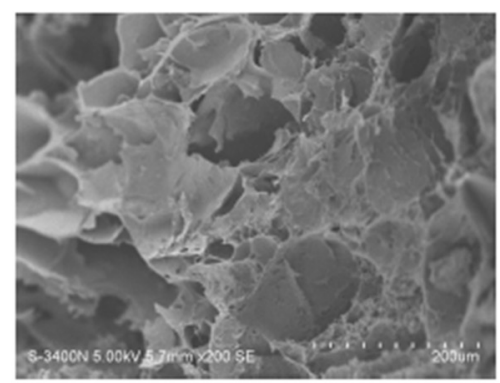

e

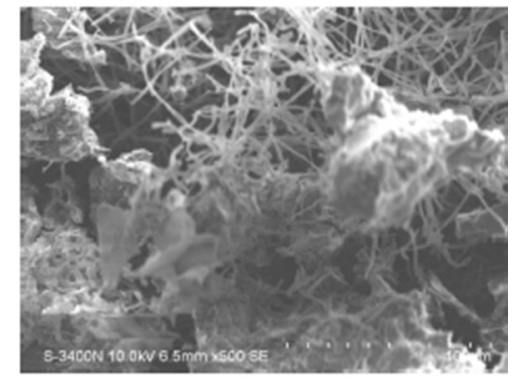

C

Table 1. Gel formulation prepared in the study.

\begin{tabular}{|c|c|c|c|c|c|c|}
\hline \multicolumn{2}{|c|}{ Gel formulation * } & $\begin{array}{c}\text { Chitosan/Vitamin } \\
\text { C }(5: 1)(w / w \%) \\
\end{array}$ & $\begin{array}{l}\text { Nystatin } \\
(w / w \%)\end{array}$ & $\begin{array}{l}\text { Krill oil } \\
(w / w \%)\end{array}$ & $\begin{array}{c}\text { Hydroxyapatite } \\
(w / w \%)\end{array}$ & pH \\
\hline $\mathrm{Ch} / \mathrm{Vit} \mathrm{C}$ & Gel 1 & 5 & 0 & 0 & 0 & 5.46 \\
\hline $\mathrm{Ch} /$ Vit C/Nyst & Gel 2 & 5 & 1 & 0 & 0 & 6.84 \\
\hline $\mathrm{Ch} / \mathrm{Vit} \mathrm{C} / \mathrm{HA} / \mathrm{Nyst}$ & Gel 3 & 5 & 1 & 0 & 1 & 6.74 \\
\hline $\mathrm{Ch} / \mathrm{Vit} \mathrm{C} / \mathrm{Nyst} / \mathrm{Kr}$ & Gel 4 & 5 & 1 & 1 & 0 & 6.94 \\
\hline $\mathrm{Ch} / \mathrm{Vit} \mathrm{C} / \mathrm{Nyst} / \mathrm{Kr} / \mathrm{HA}$ & Gel 5 & 5 & 1 & 1 & 1 & 6.65 \\
\hline
\end{tabular}

* Ch, Chitosan; Vit C, vitamin C; Nyst, nystatin; HA, hydroxyapatite; Kr, krill oil.

The cumulative in vitro release of nystatin from the hydrogels, directly after manufacture and after three-month storage, is shown in Figures 2 and 3, respectively.

The in vitro release of therapeutic agents from the newly prepared hydrogel was carried out using USP dissolution apparatus type I. As the regression analysis of the obtained results for two kinetic models including zero order and Higushi's model showed that Higushi's model gave the highest value of $r^{2}$ with significant difference $(p<0.05)$. Higushi's model, where the cumulative amount of the released drug per unit area is proportional to the square root of time, is the more suitable model to describe the release kinetics from the gel preparations examined in the present study. The release of therapeutic agents from the hydrogels was studied as demonstrated in Figures 2 and 3. The release of the corresponding hydrogels containing nystatin as a potential therapeutic agent prototype remained stable in the early hours of the experiment, allowing a more constant release, which would ensure an effective and prolonged anti-microbial activity when applied clinically. This property would make the system a suitable candidate for further development as a functional dual action restorative material. 
Figure 2. Cumulative percentage release of nystatin directly after manufacturing of the different hydrogels $(n=3, p<0.05)$. Ch, Chitosan; Vit C, vitamin C; Nyst, nystatin; HA, hydroxyapatite; Kr, krill oil.

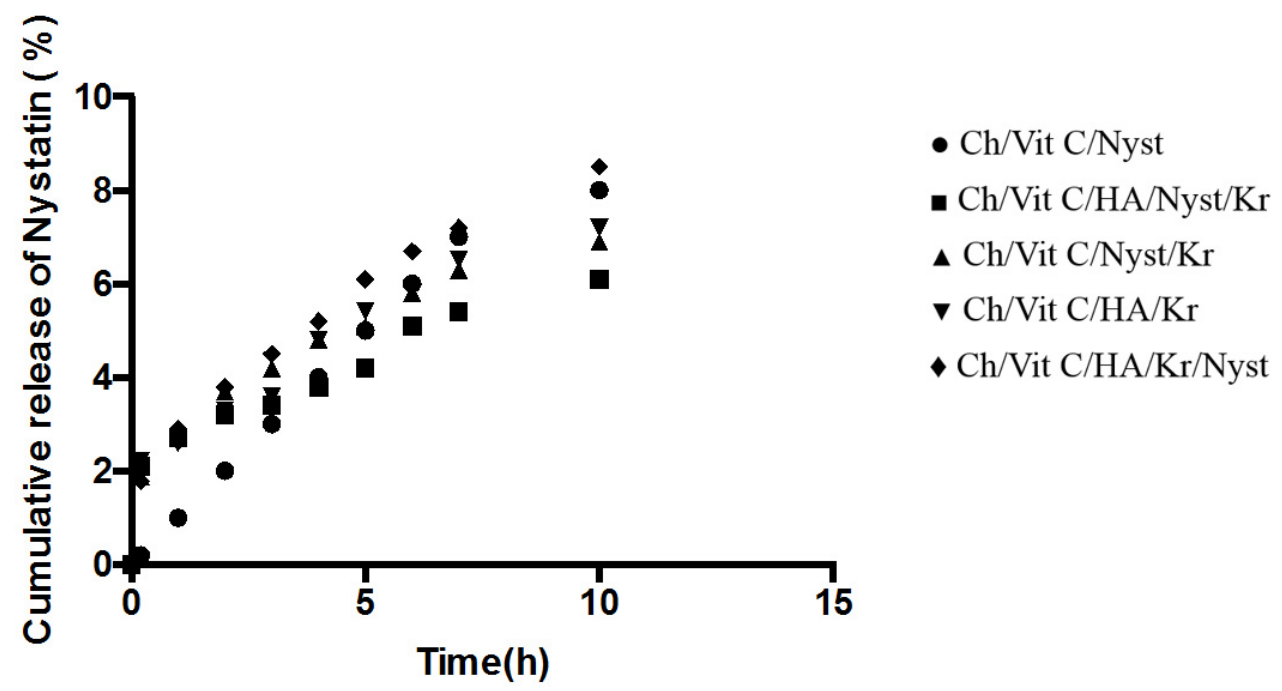

Figure 3. Percentage release of nystatin from the different hydrogels after 3 months storage at $21{ }^{\circ} \mathrm{C}(n=3, p<0.05)$.

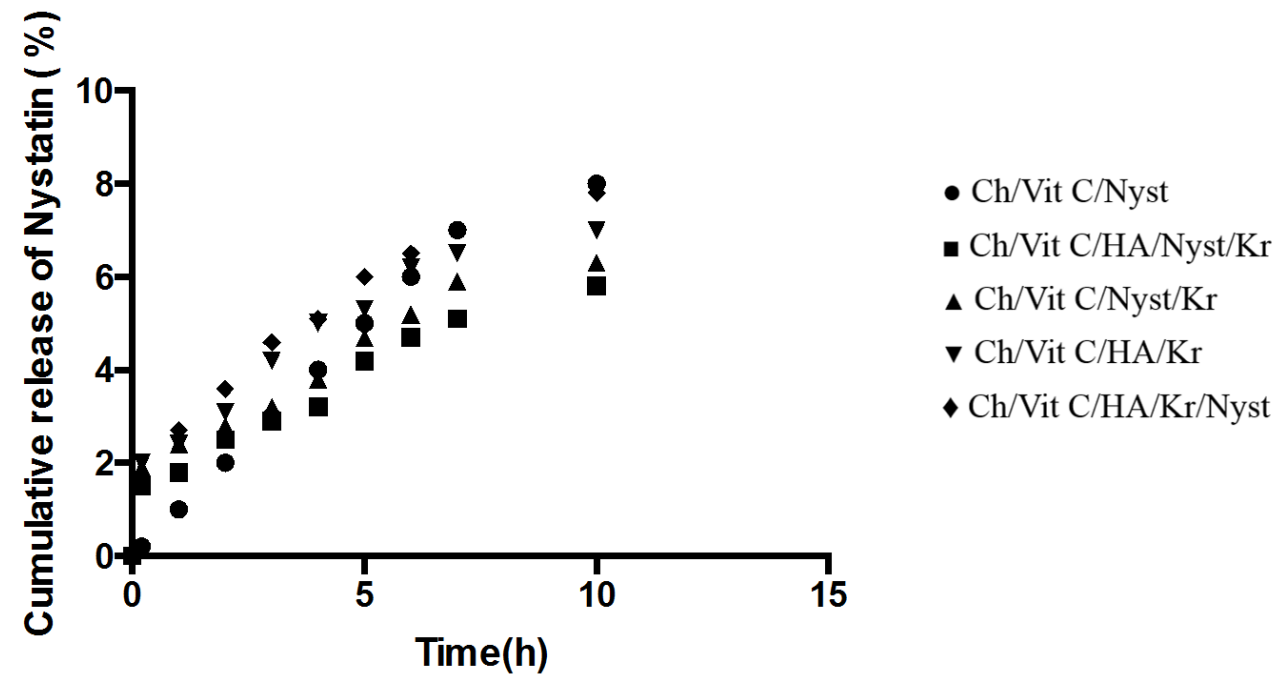

\subsection{Gel Stability}

The results suggest that there is no significant decomposition observed after six-month storage at room temperature $\left(21^{\circ} \mathrm{C}\right)$, as antioxidant capacity of the materials stored for six months showed no diminished capacity compared to the freshly prepared hydrogels.

\subsection{Studies of Equilibrium Swelling in the Hydrogels}

The hydrogels remained in the cylindrical form after swelling (Figure 4). Compared with dry state hydrogels, the swollen state hydrogel volume displayed significant increases. 
Figure 4. Swelling ratio (\%) of the investigated hydrogels.

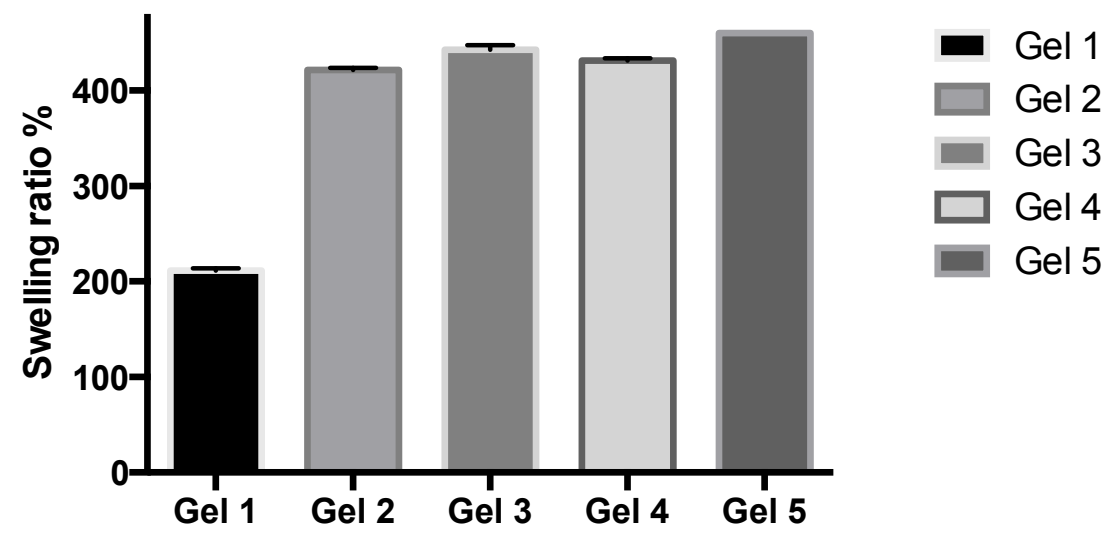

\subsection{Free Radical Defense Capability of the Prepared Hydrogels.}

When a wound occurs, it is generally accompanied by classical symptoms of inflammation, such as pain, redness and edema. In the inflammation stage, the main aim is the removal of debris, damage tissue, and bacteria by neutrophils and macrophages. These cells have a role in antimicrobial defense and debridement of devitalized tissue by production of proteolytic enzymes and reactive oxygen species [21]. The amount of uncontrolled ROS is the main cause of the inability of the healing process to continue and therefore it would be ideal to utilize the antioxidant capacity of the "designer" hydrogels to detect and fight the free radical excess. This has been assessed using a previously established model known as the biologic Fenton reaction through which the $\mathrm{HO}^{\bullet}$ free radical can be generated in the presence of $\mathrm{H}_{2} \mathrm{O}_{2}$ [22].

As reported earlier, protein cross-linking can be used as a model for detection of free radical activity and activation of "molecular defense forces" [23]. Bovine serum albumin (BSA), a completely water-soluble protein, can be polymerized by hydroxyl radicals generated by the Fenton reaction system of $\mathrm{Fe}^{2+} / \mathrm{EDTA} / \mathrm{H}_{2} \mathrm{O}_{2} /$ ascorbate. As a result, the protein loses its water-solubility and the polymerized product precipitates. The decrease in the concentration of the water-soluble protein can subsequently be detected [23].

We considered it worthwhile to study our "dual action bioactive restorative materials" as a "built-in defense mechanism" for the "site specific" counter reaction of the generated free radical production in vitro. Therefore we adopted the above-mentioned method for recording changes in water solubility of the BSA exposed to free radicals generated by an inorganic chemical system. As clearly demonstrated by Figure 5, upon exposure to standard $\mathrm{H}_{2} \mathrm{O}_{2}$ in the form of a Fe ${ }^{2+} / E D T A / \mathrm{H}_{2} \mathrm{O}_{2} /$ ascorbate solution, which served as a base line for the determination of free radical generation under "prototype in vitro free radical damage", upon incorporation of the chitosan substituted hydrogels, the built-in antioxidant capacity and therefore free radical defense of the in vitro model has been activated. This model represents the practical approach of in situ monitoring and testing of the amount of free radical production and synergistic antioxidant defense of the system. Further investigations and fine-tuning of the system are currently underway in our laboratory. 
Figure 5. Insolubility of BSA exposed to the Fenton reaction system of $\mathrm{Fe}^{2+} / \mathrm{EDTA} / \mathrm{H}_{2} \mathrm{O}_{2} /$ ascorbate in the presence of the designer hydrogels in vitro free radical detection/defense prototype system in action $(n=3, p=0.01)$.

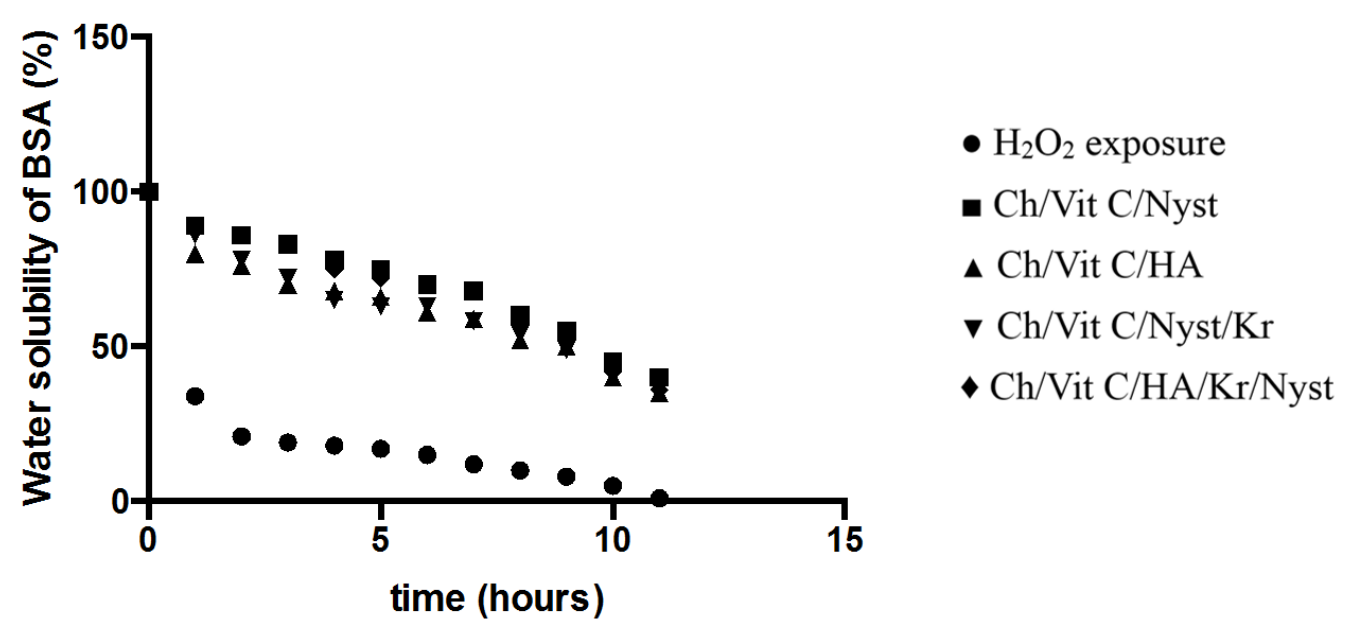

\subsection{In Vitro Antifungal Activity of Novel Nystatin/Antioxidant Containing Chitosan Hydrogels}

Although fungi are not primarily involved in the development of oral mucositis, they account for the most frequent infections of the damaged oral mucosa in immune-suppressed patients. Candidiasis is the predominant fungal infection manifesting itself by characteristic white coats or erythematous lesions in the corners of the mouth and on the soft palate and tongue [2]. In the present study, nystatin was selected because of its wide application, both locally and systemically, in treatment of oral fungal infections.

Chitosan hydrogen scaffolds were designed in this study as carriers for antibiotics and showed a steady release of the medication. Three-dimensional chitosan matrices have been shown to be excellent tissue engineering scaffolds for cell attachment and growth. Chitosan has a scalloped structure and has been used in tissue engineering to culture hepatocytes, fibroblasts and cartilage cells because of its ability to promote cell attachment and growth [24-28]. In our investigation, chitosan was selected as the carrier for nystatin, mainly because it can both carry and deliver the medication, but also because it has other useful bioactivities such as antioxidant and anti-inflammatory properties [29].

Discs with additives but without nystatin gave no inhibition zones. The different preparations containing nystatin all gave clear inhibition zones with significant difference amongst them (Table 2). Furthermore, the test samples with nystatin and with additives all gave a significantly smaller inhibition zone than the nystatin antibiotic control disc although they contained nystatin at a higher concentration (Figure 6). This indicates that release of the nystatin from the formulations was inhibited to some extent, which is in accordance with previous results obtained from the nystatin release studies that showed that the cross-linked chitosan sponges were able to deliver active antibiotic for up to 10 days [16]. This slow release of the nystatin from the gel will be a beneficial effect that may enable a sustainable release over time. 
Table 2. Diameters of Nystatin inhibition zones.

\begin{tabular}{lcc}
\hline \multirow{2}{*}{ Sample } & \multicolumn{2}{c}{ Diameters $(\mathbf{m m})$} \\
\cline { 2 - 3 } & Average $(\boldsymbol{n}=\mathbf{3})$ & STD \\
\hline Nystatin & 20.17 & 0.3 \\
$\mathrm{Ch} /$ Vit C/Nyst & 10.08 & 0.23 \\
$\mathrm{Ch} / \mathrm{Vit} \mathrm{C} / \mathrm{HA} / \mathrm{Nyst}$ & 9.31 & 0.34 \\
$\mathrm{Ch} / \mathrm{Vit} \mathrm{C} / \mathrm{HA} / \mathrm{Nyst} / \mathrm{Kr}$ & 8.36 & 0.33 \\
$\mathrm{Ch} / \mathrm{Vit} \mathrm{C} / \mathrm{HA} / \mathrm{Nyst} / \mathrm{Kr} / \mathrm{Fe}^{2+}$ & 7.84 & 0.52 \\
$\mathrm{Ch} / \mathrm{Vit} \mathrm{C} / \mathrm{HA} / \mathrm{Nyst} / \mathrm{Fe}^{2+}$ & 8.91 & 0.33 \\
\hline
\end{tabular}

Figure 6. Example of C. albicans growth inhibition zones produced by the hydrogels tested.

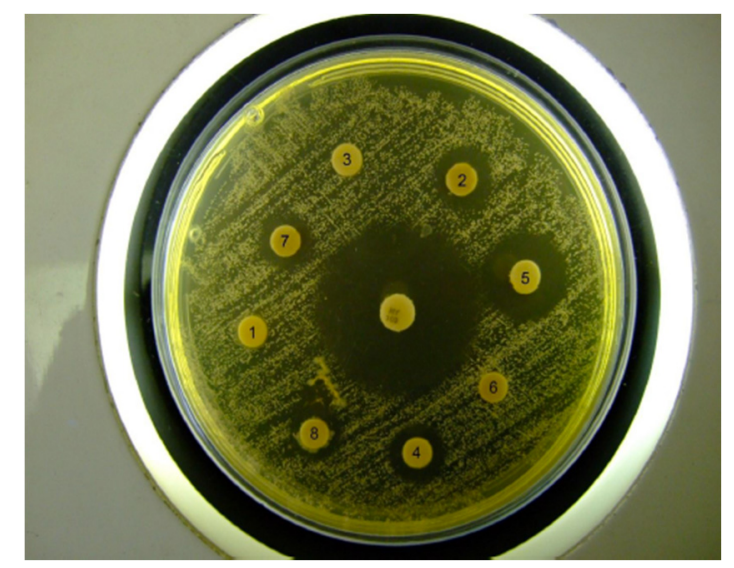

\subsection{Bio-Adhesion in Vitro Model}

The term bio-adhesion refers to any bond formed between two biological surfaces or between a biological and a synthetic surface. In case of bio-adhesive drug delivery, the term bio-adhesion is used to describe the adhesion between polymers, either synthetic or natural, and soft tissues or the gastrointestinal mucosa [30]. In cases where the bond is formed with the mucus, the term muco-adhesion may be used synonymously with bio-adhesion [30]. Muco-adhesion can therefore be defined as a state in which two components, of which one is of biological origin, are held together for extended periods of time by the help of interfacial forces. Generally speaking, bio-adhesion is a term, which broadly includes adhesive interactions with any biological or biologically derived substance, and the term muco-adhesion is used when the bond is formed with a mucosal surface [30].

Over the last two decades, chitosan has been used for various biomedical and drug delivery applications due to its low toxicity, good biocompatibility and anti-microbial and bio-adhesive properties [31].

Higher adhesiveness of the gels is desired to maintain an intimate contact with the oral mucosa and preliminary in vitro results on the model adhesive surface (band-aid used as a prototype system) are summarized in Table 3. Chitosan hydrogels showed high adhesive force and this work of adhesion can be expected because of the well-known intrinsic bio-adhesive properties of chitosan. The adequate water absorption capacity together with the cationic nature, which promotes binding to the negative surface of the mucosa, can also explain these results [31]. 
According to Caffaggi, hydration of the polymer causes mobilization of the polymer chains and hence influences polymeric adhesion [32]. Appropriate swelling is important to guarantee adhesion, however, over hydration can form slippery non-adhesive hydrogels [33]. In addition the molecular arrangement of the polymeric chains, which are present in the new hydrogels with additives, such as nystatin, Krill oil, Vitamin C and hydroxyapatite, can further enable the hydrogel to interact with the substrate [31]. The correlation between the force and work of adhesion is noticeable for all. Further experiments are to be conducted to evaluate the bio-adhesive capacity of the designer hydrogels.

Table 3. Bio-adhesion testing in vitro. The presented values are an average $(n=5)$.

\begin{tabular}{ccc}
\hline Hydrogel & Adhesive force $(\mathbf{N}) \pm$ SD & Work of adhesion $(\mathbf{N} \cdot \mathbf{c m}) \pm \mathbf{S D}$ \\
\hline Gel 1 & $1.20 \pm 0.30$ & $3.35 \pm 0.48$ \\
Gel 2 & $1.12 \pm 0.27$ & $3.19 \pm 0.52$ \\
Gel 3 & $1.01 \pm 0.30$ & $2.85 \pm 0.41$ \\
Gel 4 & $1.15 \pm 0.40$ & $3.31 \pm 0.31$ \\
\hline
\end{tabular}

\subsection{Shear Bond Strength Tests for Dentin Bonding}

Mean shear bond strength values and difference between the groups are summarized in Figure 7 for bonding to dentin after 24 hours. In general there was an increase in bond strength of the dentin treated with the antioxidant (chitosan:Vit $\mathrm{C}$ complex) containing hydrogels compared to the bond strength of the conventionally bonded teeth. Interestingly, the increase in bond strength was also observed in the groups of hydrogen peroxide exposed samples and with no primer (conventional self etching bonding system commonly employed in restorative dentistry) used in the bonding exercises, suggesting that there additional benefits associated with chitosan:antioxidant system are in need of further investigations [31].

Figure 7. Shear bond strength of hydrogels after 24 hours of bonding to dentin, where Group A and Group B represent negative and positive control in the experiments, as described in Table 4

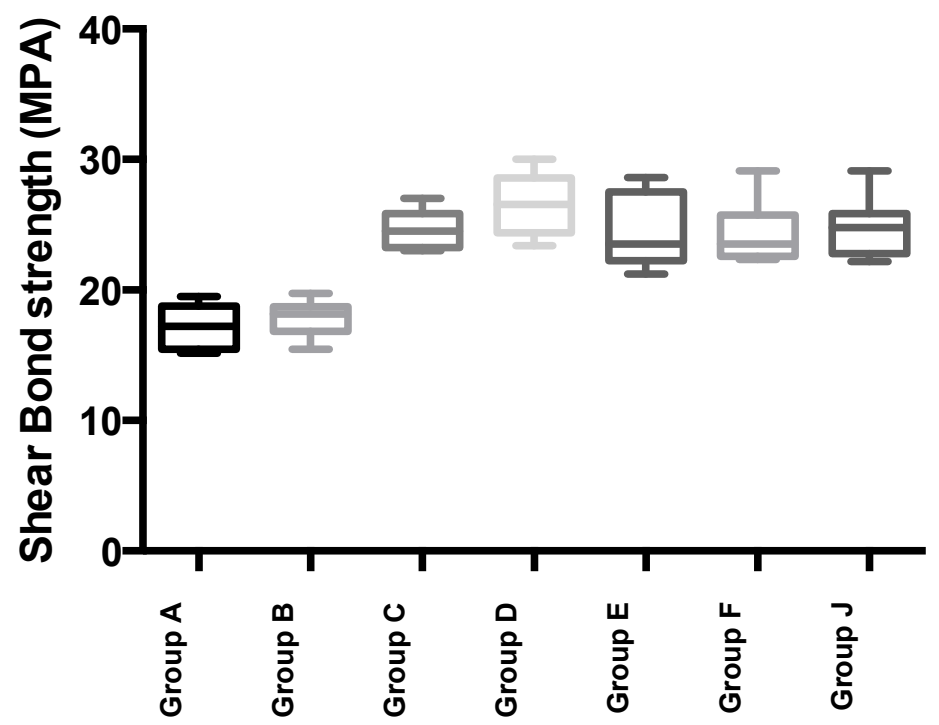


The results of this study suggests that the optimum results for the strengthening of dentin can be achieved throughout the immediate treatment with chitosan:nystatin "host:guest" complex with the increase of dentin bond strength. The additional advantage of the system may suggest that, antioxidant release from chitosan gel depends on the physical host:guest structure as well as $\mathrm{pH}$ properties and flexibilities of the material [32-34]. The additional benefit of using chitosan:nystatin:antioxidant system as a bonding/pre-bonding to enamel and dentin system lies in its ability to show favorable immediate results in terms of bonding effectiveness[27,34-40]. Therefore, the newly developed chitosan:antioxidant (combination of vitamin $\mathrm{C}$ and krill oil) systems, supporting our earlier reported results [27], are able to address the shortfalls affecting the long-term bonding performance of modern adhesives and addresses the current perspectives for improving bond durability of conventional adhesive systems.

\section{Experimental Section}

Chitosan:vitamin C (5:1) (Aldrich, Sydney, Australia), $\beta$-cyclodextrin (Aldrich, Sydney, Australia), glycerol (Sigma, Sydney, Australia), glacial acetic acid (E. Merck, Sydney, Australia) were used as received. The degree of de-acetylation of typical commercial chitosan used in this study is $87 \%$. Chitosan with molecular weight $2.5 \times 10^{3} \mathrm{KD}$ was used in the study. The isoelectric point is 4.0-5.0. Resveratrol, $\beta$-carotene and propolis (Aurora Pharmaceuticals, Melbourne, Australia) were used as bought.

\subsection{Methods}

\subsubsection{Preparation of the Various Antibiotic/Antioxidant Containing Hydrogels}

Chitosan hydrogels were prepared using the methodology previously described $[11,16]$. Briefly, the antibiotic and antioxidant powders were incorporated into the hydrogels by dispersion of $0.2 \mathrm{~g}$ of the corresponding powder in glycerol using a mortar and pestle. Ten milliliters of a $5 \%(\mathrm{w} / \mathrm{w})$ chitosan solution in glacial acetic acid was then added with continuous mixing to form the hydrogel. The strength of nystatin in the prepared gels was 100000 I.U. in each gram of the base. A summary of the newly prepared materials are highlighted in Table 1.

\subsubsection{Determination of Gel $\mathrm{pH}$}

One gram of the prepared gel was accurately weighed and dispersed in $10 \mathrm{~mL}$ of distilled water. The $\mathrm{pH}$ of the dispersions was measured using a standard $\mathrm{pH}$ meter (HANNA instruments, HI8417, HANNA, Keysborough, Australia).

\subsubsection{In Vitro Nystatin Release}

The release of nystatin from the gels was carried out with a United States Pharmacopeia (USP) dissolution apparatus type 1 (Copley Scientific, London, UK). In order to overcome the small volume of the dissolution medium, $100 \mathrm{~mL}$ beakers were used instead of the supplied jars. The basket of the dissolution apparatus was filled with $1 \mathrm{~g}$ of nystatin gel on a filter paper. The basket was immersed to about $1 \mathrm{~cm}$ of its surface in $50 \mathrm{~mL}$ of phosphate buffer at pH 6.8 and stirred at $100 \mathrm{rpm}$ at $37 \pm 0.5^{\circ} \mathrm{C}$. Twenty four samples of $2 \mathrm{~mL}$ each were collected at $0.2,1,2,3,4,5,6,7,8,10,15$ and $24 \mathrm{~h}$ and the 
nystatin concentration in the samples was determined with a Ultraviolet (UV) spectrophotometer (Cintra 5, GBC Scientific Equipment, Melbourne, Australia) at a wavelength of $306 \mathrm{~nm}$. Three replicate measurements were performed for each formulation. During the sampling process each sample volume was replaced by the same volume of phosphate buffer at $\mathrm{pH} 6.8$ to maintain a constant volume and sink condition.

\subsubsection{Microbiological Investigations}

Candida albicans strain NCPF 3153 (National Collection of Pathogenic Fungi, UK) was used as test organism. The antifungal effectiveness of the prepared gels was measured using the standard Kirby-Bauer agar diffusion method [17]. Muller-Hinton agar (Oxoid, London, UK) plates were inoculated by streaking a standardized inoculum containing $10^{7}-10^{8} \mathrm{CFU}$ with a cotton swab. $5 \mathrm{mg}$ of each hydrogel was applied to $6 \mathrm{~mm}$ diameter paper discs (500 I.U./disc). The paper discs were placed on the Muller-Hinton agar medium and incubated at $37{ }^{\circ} \mathrm{C}$ for $24 \mathrm{~h}$. The effectiveness of the prepared gels was compared to chitosan gel containing no nystatin and an antibiotic sensitivity disc (Mast Laboratories, Merseyside, UK) containing 100 I.U. of nystatin/disc. The diameter of the zones of growth inhibition was measured from 3 different angles with a caliper. Each combination of additive was tested in triplicate.

\subsubsection{Bio-Adhesive Investigation}

Bio-adhesion studies were done using a Chatillon apparatus (Chatillon, Ametek, Largo, FL, USA) for force measurement. This method determines the maximum force and work needed to separate two surfaces in intimate contact. The hydrogels $(0.1 \mathrm{~g})$ were homogeneously spread on a $1 \mathrm{~cm}^{2}$ glass disc and then the discs were fixed to the support of the tensile strength tester using double side adhesive. The gel was brought into contact with a commercially available Band-Aid strip in order to simulate skin attachment. After a preset contact time of 1 min under contact strength of $0.5 \mathrm{~N}$, the 2 surfaces were separated at a constant rate of displacement at $1 \mathrm{~mm} / \mathrm{s}$. The strength was recorded as a function of the displacement, which allowed determination of the maximal detachment force $F_{\max }$ and the work of adhesion $W$, which was calculated from the area under the strength-displacement curve $[18,19]$.

\subsubsection{Morphology of the Gels}

The samples were prepared by freezing in liquid nitrogen for $10 \mathrm{~min}$, and then freeze-dried for $24 \mathrm{~h}$. The prepared samples were fractured in liquid nitrogen using a razor blade. The fractured samples were dried under a vacuum, attached to metal stubs, and sputter coated with gold under a vacuum for the SEM study. The interior and the surface morphology were observed under a scanning electron microscope (SEM, Hitachi S4800, Tokyo, Japan).

\subsubsection{Gel Stability}

Stability of the gel formulations was also investigated. The organoleptic properties (color, odor), $\mathrm{pH}$, drug content, and release profiles of the gels stored at $20{ }^{\circ} \mathrm{C}$ were examined on days $0,15,30$ and 178 . 
The performance of the hydrogels was not affected by the storage conditions, suggesting remarkable stability of the novel biomaterials under investigation.

\subsubsection{Studies of Equilibrium Swelling in the Hydrogels}

A known weight of the dry gel was placed in a tea infusion bag and immersed in $\mathrm{pH} 4.0$ and $\mathrm{pH} 9.0$ buffer solutions, respectively, and kept at $25^{\circ} \mathrm{C}$ for $48 \mathrm{~h}$ until equilibrium of swelling had been reached. The swollen gels were taken out and immediately weighed with a microbalance after the excess water on the surfaces was absorbed with a filter paper. The equilibrium swelling ratio (SR) was calculated using the following equation:

$$
\operatorname{SR}(\%)=\left(W_{\mathrm{s}}-W_{\mathrm{d}}\right) / W_{\mathrm{d}} \times 100
$$

where $W_{\mathrm{s}}$ and $W_{\mathrm{d}}$ are the weights of the gels at the equilibrium swelling state and at the dry state, respectively [20]. Experiments were repeated in triplicate for each gel specimen and the mean value calculated.

\subsubsection{Shear Bond Strength Tests for Dentin Bonding}

Extracted non-carious, intact, human molars stored in water containing a few crystals of thymol at $4{ }^{\circ} \mathrm{C}$ were used within two months using protocol previously described by US [21-23]. 56 teeth samples prepared and divided into 7 groups of 8 each, A-F (Table 4) and stored in a solution of artificial saliva. These groups were then treated as outlined in Table 4. After 24 hours, a stud of each tooth was tested for shear bond strength. An Instron Universal Testing Machine at a crosshead speed of $0.5 \mathrm{~mm} / \mathrm{min}$ was used to test the de-bonding strength. All data tests were analyzed using the non-parametric ANOVA test.

Table 4. Groups tested (8 teeth per groups).

\begin{tabular}{lc}
\hline Samples & Conditions \\
\hline Group A & $37 \%$ of phosphoric acid + primer+ Bonding immediately (negative control) \\
Group B & Self-etching primer + Bonding immediately (positive control) \\
Group C & Gel 1 + Bonding immediately \\
Group D & Gel 2 + Bonding immediately \\
Group E & Gel 3 + Bonding immediately \\
Group F & Gel 4 + Bonding immediately \\
Group J & Gel 5 + Bonding immediately \\
\hline
\end{tabular}

\section{Conclusions}

We evaluate the novel chitosan based functional drug delivery systems which can be successfully incorporated into "dual action bioactive restorative materials" capable to induce in vitro improved wound healing prototype and containing an antibiotic such as nystatin, krill oil as an antioxidant and hydroxyapatite as a molecular bone scaffold as function specific biomaterials capable of initiating bio-repair and bio-adhesion in vitro in a site specific manner. 


\section{Author Contributions}

V. Tamara Perchyonok and Sias Grobler conceived and designed the experiments; V. Tamara Perchyonok, Shengmiao Zhang and Nicholas Basson performed the experiments; V. Tamara Perchyonok, Vanessa Reher and Sias Grobler analyzed the data; Shengmiao Zhang and Nicholas Basson contributed reagents/materials/analysis tools; V. Tamara Perchyonok wrote the paper.

\section{Conflicts of Interest}

The authors declare no conflict of interest.

\section{References}

1. Peterson, D.E. Research advances in oral mucositis. Curr. Opin. Oncol. 1999, 11, 261-266.

2. Kostler, W.J.; Hejna, M.; Wenzel, C.; Zielinski, C.C. Oral mucositis complicating chemotherapy and/or radiotherapy: Options for prevention and treatment. CA Cancer J. Clin. 2001, 51, 290-315.

3. Naidu, M.U.; Ramana, G.V.; Rani, P.U.; Mohan, I.K.; Suman, A.; Roy, P. Chemotherapy-induced and/or radiation therapy-induced oral mucositis - Complicating the treatment of cancer. Neoplasia 2004, 6, 423-431.

4. Sonis, S.T. Mucositis as a biological process: A new hypothesis for the development of chemotherapy-induced stomatotoxicity. Oral Oncol. 1998, 34, 39-43.

5. Pham-Huy, L.; He, H.; Pham-Huy, C. Free radicals, antioxidants in diease and health. Int. J. Biomed. Sci. 2008, 4, 89-96.

6. Valko, M.; Leibfritz, D.; Moncol, J.; Cronin, M.T.; Mazur, M.; Telser, J. Free radicals and antioxidants in normal physiological functions and human disease. Int. J. Biochem. Cell Biol. 2007, $39,44-84$.

7. Senel, S.; Kremer, M.J.; Kas, S.; Wertz, P.W.; Hincal, A.A.; Squier, C.A. Enhancing effect of chitosan on peptide drug delivery across buccal mucosa. Biomaterials 2000, 21, 2067-2071.

8. Akncbay, H.; Senel, S.; Ay, Z.Y. Application of chitosan gel in the treatment of chronic periodontitis. J. Biomed. Mater. Res. B Appl. Biomater. 2007, 80, 290-296.

9. Perchyonok, V.T.; Zhang, S.; Oberholzer, T. Alternative chitosan based drug delivery system to fight oral mucositis: Synergy of conventional and bioactives towards the optimal solution. Curr. Nanosci. 2012, 8, 541-547.

10. Aksungur, P.; Sungur, A.; Unal, S.; Iskit, A.B.; Squier, C.A.; Senel, S. Chitosan delivery systems for the treatment of oral mucositis: In vitro and in vivo studies. J. Control Release 2004, 98, 269-279.

11. Perchyonok, V.T.; Zhang, S.; Oberholzer, T. Novel melatonin-chitosan hydrogels as suitable oral bio-drug delivery systems to fight oral mucositis: Synergy of antioxidants and bioactives in action. Curr. Org. Chem. 2012, 16, 2430-2436.

12. Teng, S.H.; Lee, E.J.; Wang, P.; Jun, S.H.; Han, C.M.; Kim, H.E. Functionally gradient chitosan/hydroxyapatite composite scaffolds for controlled drug release. J. Biomed. Mater. Res. B Appl. Biomater. 2009, 90, 275-282. 
13. Boateng, J.S.; Matthews, K.H.; Stevens, H.N.; Eccleston, G.M. Wound healing dressings and drug delivery systems: A review. J. Pharm. Sci. 2008, 97, 2892-2923.

14. Majeed, A.; Al Naimi, R. Role of hydroxyapatite in healing of experimentally induced cutaneous wound in rabbits. Al-Anbar J. Vet. Sci. 2012, 5, 74-81.

15. Kim, H.W.; Knowles, J.C.; Kim, H.E. Hydroxyapatite porous scaffold engineered with biological polymer hybrid coating for antibiotic vancomycin release. J. Mater. Sci. Mater. Med. 2005, 16, 189-195.

16. Perchyonok, V.T.; Zhang, S.; Oberholzer, T. Chitosan and gelatin based prototype delivery systems for the treatment of oral mucositis: From material to performance in vitro. Curr. Drug. Deliv. 2013, 10, 144-150.

17. Bauer, A.; Kirby, W.; Sherris, J.; Turck, M. Antibiotic susceptibility testing by a standardized single disc method. Am. J. Clin. Pathol. 1966, 36, 493-496.

18. Koffi, A.A.; Agnely, F.; Ponchel, G.; Grossiord, J.L. Modulation of the rheological and mucoadhesive properties of thermosensitive poloxamer-based hydrogels intended for the rectal administration of quinine. Eur. J. Pharm. Sci. 2006, 27, 328-335.

19. Ponchel, G.; Touchard, F.; DuchQne, D.; Peppas, N. Bioadhesive analysis of controlled-release systems. I. Fracture and interpenetration analysis in poly (acrylic acid)-containing systems. J. Control. Release 1987, 5, 129-141.

20. Nair, A.B.; Kumria, R.; Harsha, S.; Attimarad, M.; Al-Dhubiab, B.E.; Alhaider, I.A. In vitro techniques to evaluate buccal films. J. Control. Release 2013, 166, 10-21.

21. Chapple, I.L. Reactive oxygen species and antioxidants in inflammatory diseases. J. Clin. Periodontol. 1997, 24, 287-296.

22. Fenton, H.J.H. LXXIII.-Oxidation of tartaric acid in presence of iron. J. Chem. Soc. Trans. 1894, 65, 899-910.

23. Kyselova, Z.; Rackova, L.; Stefek, M. Pyridoindole antioxidant stobadine protected bovine serum albumin against the hydroxyl radical mediated cross-linking in vitro. Arch. Gerontol. Geriatr. 2003, 36, 221-229.

24. Kawase, M.; Michibayashi, N.; Nakashima, Y.; Kurikawa, N.; Yagi, K.; Mizoguchi, T. Application of glutaraldehyde-crosslinked chitosan as a scaffold for hepatocyte attachment. Biol. Pharm. Bull. 1997, 20, 708-710.

25. Guo, J.F.; Jourdian, G.W.; MacCallum, D.K. Culture and growth characteristics of chondrocytes encapsulated in alginate beads. Connect. Tissue Res. 1989, 19, 277-297.

26. Zhao, F.; Yin, Y.; Lu, W.W.; Leong, J.C.; Zhang, W.; Zhang, J.; Zhang, M.; Yao, K. Preparation and histological evaluation of biomimetic three-dimensional hydroxyapatite/chitosan-gelatin network composite scaffolds. Biomaterials 2002, 23, 3227-3234.

27. Koyano, T.; Minoura, N.; Nagura, M.; Kobayashi, K. Attachment and growth of cultured fibroblast cells on PVA/chitosan-blended hydrogels. J. Biomed. Mater. Res. 1998, 39, 486-490.

28. Lee, Y.M.; Park, Y.J.; Lee, S.J. Tissue engineered bone formation using chitosan/tricalcium phosphate sponges. J. Periodontol. 2000, 71, 410-417.

29. Xia, W.; Liu, P.; Zhang, J.; Chen, J. Biological activities of chitosan and chitooligosaccharides. Food Hydrocoll. 2011, 25, 170-179. 
30. Pratima, N.; Tiwari, S.; Kamble, S. Mucoadhesive: As oral controlled gastroretentive drug delivery system. Int. J. Res. Pharm. Sci. 2012, 2, 32-59.

31. Sogias, I.A.; Williams, A.C.; Khutoryanskiy, V.V. Why is chitosan mucoadhesive? Biomacromolecules 2008, 9, 1837-1842.

32. Dodou, D.; Breedveld, P.; Wieringa, P.A. Mucoadhesives in the gastrointestinal tract: Revisiting the literature for novel applications. Eur. J. Pharm. Biopharm. 2005, 60, 1-16.

33. Patel, V.F.; Liu, F.; Brown, M.B. Advances in oral transmucosal drug delivery. J. Control. Release 2011, 153, 106-116.

34. Williams, S. Nutrition and Diet Therapy; Mosby-Year Book Inc.: St Louis, MO, USA, 1997.

35. Senel, S.; Ikinci, G.; Kas, S.; Yousefi-Rad, A.; Sargon, M.F.; Hincal, A.A. Chitosan films and hydrogels of chlorhexidine gluconate for oral mucosal delivery. Int. J. Pharm. 2000, 193, 197-203.

36. Deshmane, S.V.; Channawar, M.A.; Chandewar, A.V.; Joshi, U.M.; Biyani, K.R. Chitosan based sustained release mucoadhesive buccal patches containing verapamil HCL. Int. J. Pharm. Pharm. Sci. 2009, 1, 216-229.

37. Bansal, V.; Sharma, P.; Sharma, N.; Pal, O.; Malviya, R. Applications of chitosan and chitosan derivatives in drug delivery. Adv. Biol. Res. 2011, 5, 28-37.

38. Sarmento, B.; das Neves, J. Chitosan-Based Systems for Biopharmaceuticals: Delivery, Targeting and Polymer Therapeutics; John Wiley \& Sons: Weinheim, Germany, 2012.

39. Liu, N.; Park, H.J. Factors effect on the loading efficiency of vitamin c loaded chitosan-coated nanoliposomes. Colloids Surf. B Biointerfaces 2010, 76, 16-19.

40. Perchyonok, V.T.; Zhang, S.; Oberholzer, T. Protective effect of conventional antioxidant ( $\beta$-carotene, resveratrol and vitamin $\mathrm{E}$ ) in chitosan-containing hydrogels against oxidative stress and reversal of DNA double stranded breaks induced by common dental composites: In-vitro model. Open Nanosci. J. 2013, 7, 1-7.

(C) 2014 by the authors; licensee MDPI, Basel, Switzerland. This article is an open access article distributed under the terms and conditions of the Creative Commons Attribution license (http://creativecommons.org/licenses/by/4.0/). 